\title{
Controlled spatial and conformational display of immobilised bone morphogenetic protein-2 and osteopontin signalling motifs regulates osteoblast adhesion and differentiation in vitro
}

\author{
Elizabeth A Mitchell1,2, Benjamin T Chaffey², Andrew W McCaskie', Jeremy H Lakey² and Mark A Birch*1
}

\begin{abstract}
Background: The interfacial molecular mechanisms that regulate mammalian cell growth and differentiation have important implications for biotechnology (production of cells and cell products) and medicine (tissue engineering, prosthetic implants, cancer and developmental biology). We demonstrate here that engineered protein motifs can be robustly displayed to mammalian cells in vitro in a highly controlled manner using a soluble protein scaffold designed to self assemble on a gold surface.

Results: A protein was engineered to contain a C-terminal cysteine that would allow chemisorption to gold, followed by 12 amino acids that form a water soluble coil that could switch to a hydrophobic helix in the presence of alkane thiols. Bioactive motifs from either bone morphogenetic protein-2 or osteopontin were added to this scaffold protein and when assembled on a gold surface assessed for their ability to influence cell function. Data demonstrate that osteoblast adhesion and short-term responsiveness to bone morphogenetic protein- 2 is dependent on the surface density of a cell adhesive motif derived from osteopontin. Furthermore an immobilised cell interaction motif from bone morphogenetic protein supported bone formation in vitro over 28 days (in the complete absence of other osteogenic supplements). In addition, two-dimensional patterning of this ligand using a soft lithography approach resulted in the spatial control of osteogenesis.
\end{abstract}

Conclusion: These data describe an approach that allows the influence of immobilised protein ligands on cell behaviour to be dissected at the molecular level. This approach presents a durable surface that allows both short (hours or days) and long term (weeks) effects on cell activity to be assessed. This widely applicable approach can provide mechanistic insight into the contribution of immobilised ligands in the control of cell activity.

\section{Background}

It has long been recognised that cell regulatory molecules, such as growth factors and cytokines, exert powerful influences on the behaviour of eukaryotic cells at the interface of tissues. Indeed the immobilized activity of these signalling mediators in combination with the extracellular matrix (ECM) underpins many fundamental biological processes including embryo-, morpho- and tumorogenesis and wound healing [1]. Numerous studies have established the principle that tethered ligands regu-

\footnotetext{
* Correspondence: m.a.birch@ncl.ac.uk

1 Institute for Cellular Medicine, The Medical School, Newcastle University, Newcastle-upon-Tyne, NE2 4HH, UK

Full list of author information is available at the end of the article
}

( 2010 Mitchell et al; licensee BioMed Central Ltd. This is an Open Access article distributed under the terms of the Creative Commons B. Wed Central Attribution License (http://creativecommons.org/licenses/by/2.0), which permits unrestricted use, distribution, and reproduction in any medium, provided the original work is properly cited. late cell behavior quite distinctly from their freely diffusible counterpart. For example, extracellular matrix proteins like fibronectin promote adhesion [2] and migration [3] on a surface but when added exogenously to cells can have adhesion-independent effects, for example activating intracellular signalling cascades [4]. Furthermore, soluble growth factors, like fibroblast growth factor (FGF)-2, vascular endothelial growth factor (VEGF) and interleukin (IL)-1 exhibit elevated activity when presented within the context of matrix such as fibrin [5-7]. Whilst these and many other studies have provided fundamental insight into the underlying biology, the experimental approaches have meant that immobilised ligands are often presented in orientations and conformations 
that are not well controlled, at densities that are poorly characterised and under conditions in which the durability and stability of the ligand is undefined.

Our approach has been to design and fabricate protein scaffolds that can display protein ligands on self-assembled monolayers (SAM) in a controlled and reproducible way $[8,9]$. This work uses a TolAIII-fusion expression system to provide the engineered protein at high yields and solubility [10]. A cysteine residue engineered into the Cterminal end of the protein ensures durable chemisorption onto gold surfaces whilst a 12 amino acid stretch termed the switch-tag (ST) can switch from a water soluble coil to a hydrophobic helix that co-assembles with the alkane thiols of the SAM [8]. Therefore formation of a SAM containing both the scaffold protein and an amphiphile produces a surface where the orientation of the protein is controlled and non-specific interactions are low. These surfaces provide the opportunity to address how immobilised ligands can both provide insights into cell function and contribute to tissue engineering.

We demonstrate here the ability of this system to influence the long term function of cells in vitro via the incorporation of two distinct ligand motifs. The first is derived from the extracellular matrix protein, osteopontin (OPN), and was identified as supporting cell adhesion through the $\alpha_{9} \beta_{1}$ integrin [11] and subsequently shown to promote adhesion and migration of endothelial cells leading to enhanced angiogenesis [12-14]. The second is the knuckle epitope from bone morphogenetic protein (BMP)-2, a member of the transforming growth factor (TGF) $\beta$ superfamily, which has been widely shown to support commitment of cells to the osteoblastic lineage, their differentiation and functional ability to make bone $[15,16]$.

Here we show that Tol-OPN-ST dose-dependently supported the adhesion and spreading through vinculin adhesion sites of primary rat osteoblasts. Biological activity of the BMP motif within the scaffold protein was verified by adding the recombinant protein to cells transfected with a Sma- and MAD-related protein (SMAD) responsive-reporter construct and demonstrating SMAD1 activation. Tol-BMP2-ST reproduced BMP signalling and produced bone-like nodules on patterned surfaces.

This technology effectively immobilises bioactive protein motifs on a surface for analysis of cell behaviour in vitro and may provide the basis for future tissue engineering approaches.

\section{Results}

Incorporating osteopontin or BMP2 motifs in the Tol scaffold protein does not influence its binding to gold

The motifs from osteopontin and BMP2 were inserted between Tol and the Switch tag to create Tol-OP-ST and
Tol-BMP-ST respectively (Figure 1). This design ensured that the C-terminal cysteine was available to bind gold surfaces and that the biological activity of the inserted motifs is not compromised. BIAcore analysis (Table 1) shows that Tol-OP-ST and Tol-BMP-ST specifically interact with the gold surface and, interestingly, binding of Tol-OPN-ST and Tol-BMP-ST was slightly higher than Tol-ST alone. The vector includes a protease cleavage site which can remove the Tol protein leaving just OPN-ST or BMP-ST. However since the larger construct is more stable and easier to handle in solution, the initial studies used the complete fusion protein

\section{Cells specifically interact with immobilised Tol-OPN-ST}

Having demonstrated that the proteins chemisorb to the gold surface irrespective of the active motif inserted in

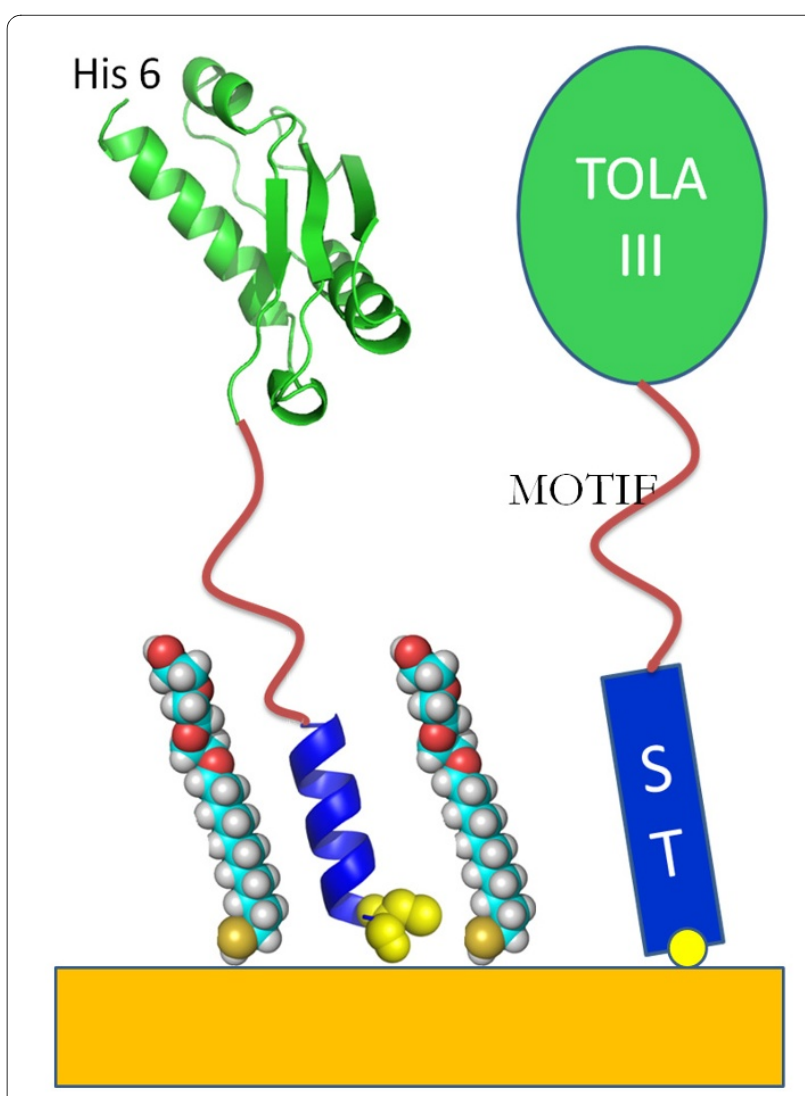

Figure 1 The design of the Tol switch tag system, a molecular image (left) and a schematic (right). The protein starts at the N-terminus with a 6 His tag for easy purification followed by a FLAG-tag sequence to aid detection. The TolAlll domain which comes next provides solubility and drives high levels of expression for fusion proteins attached to its $C$ terminus [10]. The fusion sequence is inserted between the TolA and the switch tag, which is found at the $C$ terminus, by use of a multiple cloning site in the plasmid [8]. The TolA domain can be removed by specific proteolytic cleavage but in most cases it is left in place since it stabilises the fusion construct. The switch tag is shown here as a helix whose hydrophobic properties allow co assembly with alkane thiol SAM, in solution it is a water soluble random coil. 
Table 1: SPR data of the Tol, Tol-Osteopontin (Tol-OP) and Tol-BMP proteins binding to a gold surface in SPR analysis (BIAcore).

\begin{tabular}{lcccc}
\hline Protein & rU increase & Mr of protein & moles $/ \mathbf{m m 2}$ & Molecules $/ \mathbf{m m 2}$ \\
\hline Tol & $1,526.53$ & $1,4874.4$ & $1.03 \times 10^{-13}$ & $6.4 \times 10^{10}$ \\
\hline Tol-Op & $4,381.15$ & $18,407.3$ & $2.38 \times 10^{-13}$ & $14.8 \times 10^{10}$ \\
\hline Tol-BMP & $3,162.26$ & $17,894.8$ & $1.77 \times 10^{-13}$ & $11.0 \times 10^{10}$ \\
\hline
\end{tabular}

The proteins were added at a concentration of $5 \mathrm{mM}$ in a total volume of $30 \mu \mathrm{l}$ over a period of 15 minutes.

the scaffold, the ability of the cells to interact with these immobilised proteins under serum-free conditions was determined (Figure 2a, b, c, d). After 24 hours of culture Tol-OPN-ST promoted the attachment of significant $(P<$ 0.01 ) numbers of cells compared to all other surfaces (Figure 2e). Analysis of cell morphology revealed that on TolOPN-ST, area (Figure 2f) was significantly $(P<0.05)$ enhanced compared to bare gold/11-mercapto-1-triethyleneglycolundecane (TEG-thiol) whilst circularity (Figure $2 \mathrm{~g})$ was significantly $(P<0.01)$ different from all of the other surfaces that were studied. In addition, around the periphery of the cells there were focal contacts, characterised by the identification of the protein vinculin by immunofluorescence. These contacts are important since this is where the cell interacts with the extracellular matrix (Figure 2a) with significantly $(P<0.01)$ increased abundance of vinculin in cells on Tol-OPN-ST compared to all of the other surfaces (Figure 2h). Cells on the TolOPN-ST surface also exhibited a more spread out morphology that correlates with a more robust cytoskeleton consisting of numerous actin filaments (Figure 2f, g). Relatively few cells were observed to adhere to the surfaces displaying immobilised Tol-BMP2-ST (Figure 2c, e) or Tol-green fluorescent protein (GFP) (Figure 2b, e), indeed these surfaces were little different to surfaces composed solely of the anti-adherent TEG-thiol (Figure 2d, e). Furthermore those cells that grew on Tol-BMP, Tol-GFP and control TEG alone formed fewer focal contact structures and were less well spread on the surfaces (Figure 2f, g, h).

Next we investigated cell adhesion on surfaces with varying densities of immobilised scaffold (Figure 3a, b, c). Scaffold was immobilised at different densities by adjusting the coating concentration of protein. The number of cells binding to the osteopontin surface was dependent on the surface density of Tol-OP-ST (Figure 3d) and this correlated closely with both cell area (Figure 3e) and the abundance of vinculin-containing focal contact structures (Figure 3f). Analysis of cell morphology on these surfaces demonstrates the influence of Tol-OPN-ST density on cell behaviour. At relatively low density $\left(2.1 \times 10^{7}\right.$ molecules $/ \mathrm{mm}^{2}$ ) Tol-OPN-ST surfaces promote poor cell adhesion and limited cell spreading. While at a high density $\left(1.6\right.$ and $3.3 \times 10^{10}$ molecules $\left./ \mathrm{mm}^{2}\right)$, Tol-OPN-ST supports significant cell adhesion $(P<0.01)$, extensive cell spreading $(P<0.05)$ and the formation of significantly more abundant and complex cytoskeletal elements $(P<$ $0.01)$. At an intermediate surface density $\left(2.7 \times 10^{9}\right.$ molecules $/ \mathrm{mm}^{2}$ ) (Figure $3 \mathrm{~b}$ ), cell adhesion is more weakly promoted with cellular processes extended but not sufficiently strengthened to facilitate cell spreading. To further illustrate the biological specificity of our approach we investigated the cellular response to soluble recombinant BMP2 (srBMP2) when cultured on surfaces displaying different densities of Tol-OPN-ST. It is widely appreciated that integrin-mediated adhesion influences the regulation of growth factor induced signalling cascades and indeed this phenomenon is believed to underpin numerous biological events such as morphogenesis and wound healing [17]. For this study, osteoblasts were transfected with a luciferase reporter construct driven by SMAD-binding elements and cultured on different densities of immobilised Tol-OP-ST for eight hours under serum-free conditions. The cells were then treated with soluble recombinant BMP2 and the response assessed through analysis of luciferase activity (Figure 3e). Cells that were more extensively spread on higher densities of Tol-OP-ST exhibited significantly $(P<0.01)$ higher levels of BMP-induced signalling compared to cells that were poorly spread and showing fewer adhesion complexes on low density Tol-OP-ST. This confirms that BMP receptor activation is influenced by the density of ECM ligands.

\section{Osteoblasts respond specifically to both soluble and immobilised Tol-BMP-ST}

To test the ability of Tol-BMP-ST to activate BMP-dependent intracellular signalling, it was added exogenously to cells growing on conventional tissue culture plasticware. Analysis reveals that cells transfected with SMAD-binding promoter construct were dose-dependently responsive to both soluble recombinant BMP2 and Tol-BMP-ST (Figure 4a) with the highest doses significantly different from Tol-ST alone $(P<0.01)$. The cells exhibited no 
(a)

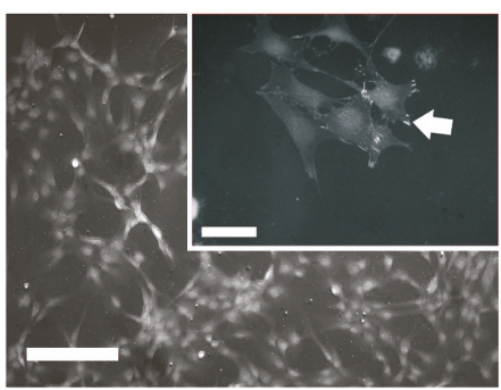

(c)

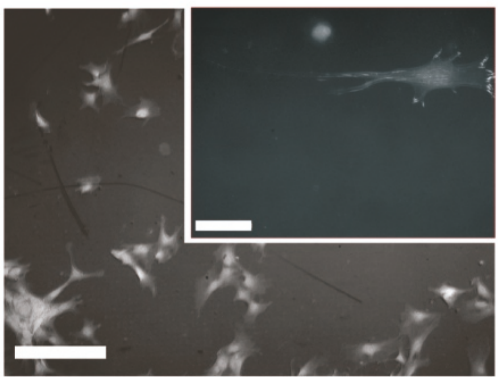

(e)

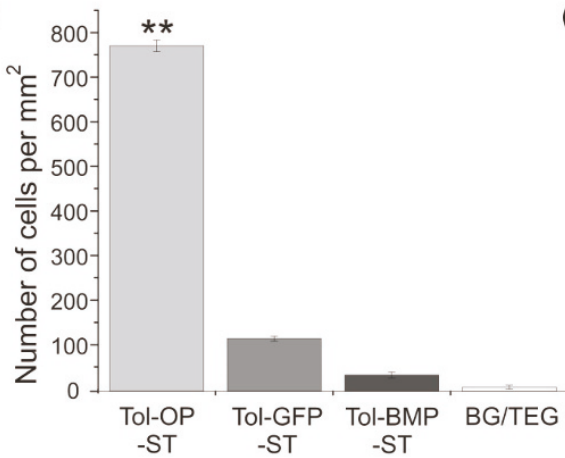

(g)

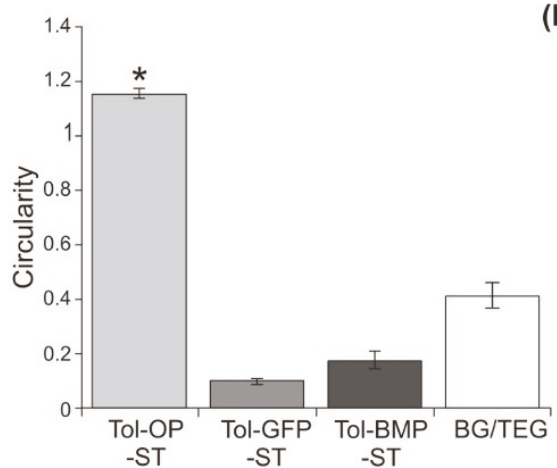

(b)

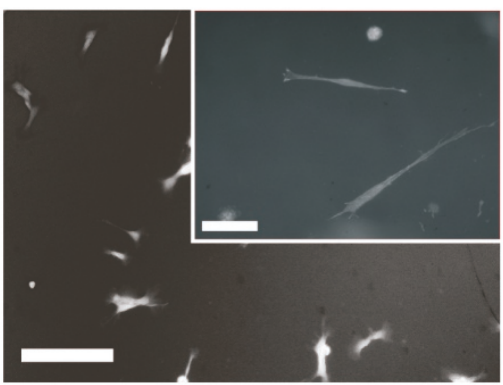

(d)
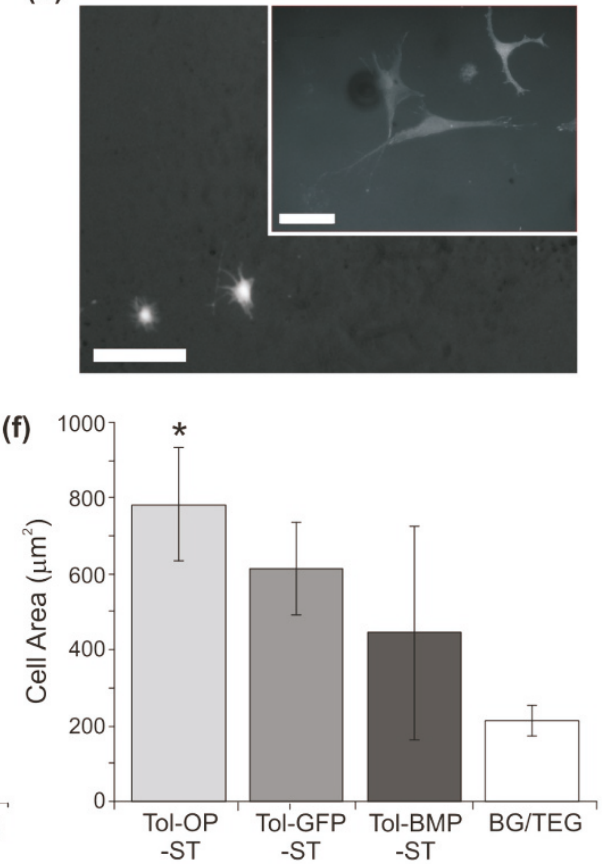

(h)

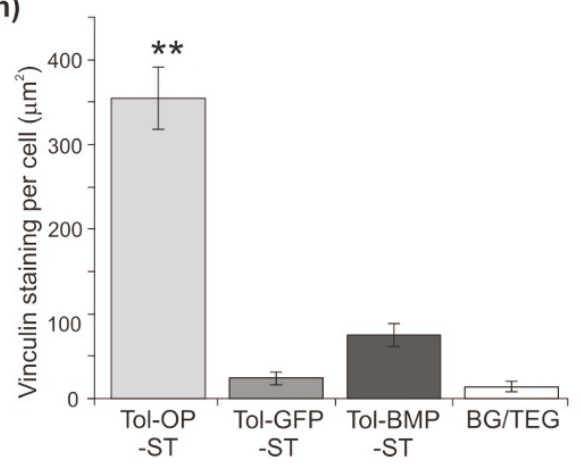

Figure 2 Immobilised Tol scaffold containing the osteopontin motif, SVVYGLR, specifically controls cell adhesion and cell morphology Cells were plated onto surfaces displaying scaffold proteins in the absence of serum supplements and assessed after 24 hours. Immunolocalization of vinculin and counterstaining with DAPI allowed identification of focal adhesion complexes (white arrow) in cells on (a) Tol-OPN-ST; (b) Tol-GFP-ST; (c) Tol-BMP-ST; (d) TEG. Size bars $=200 \mu \mathrm{m}$ and $50 \mu \mathrm{m}$ in the inset images. Image analysis demonstrated significant differences in (e) the number of cells adhered to Tol-OPN-ST compared to all other surfaces; (f) the area of cells on Tol-OPN-ST compared to TEG; $(\mathbf{g})$ the circularity of cells on Tol-OPN-ST compared to the other surfaces and (h) abundance of vinculin positivity in cells on Tol-OPN-ST compared to other surfaces. Data representative of three independent experiments; results are mean $\pm \mathrm{SEM} ;{ }^{*} P<0.05,{ }^{* *} P<0.01$. Significance was determined by ANOVA with Fisher post test. 
(a)

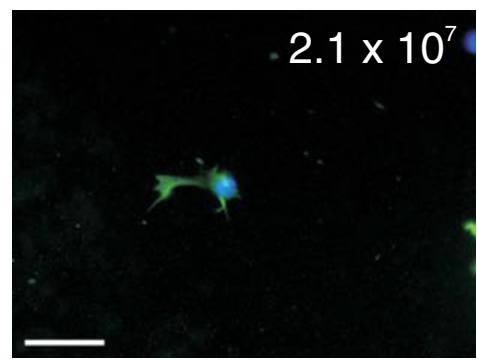

(d)

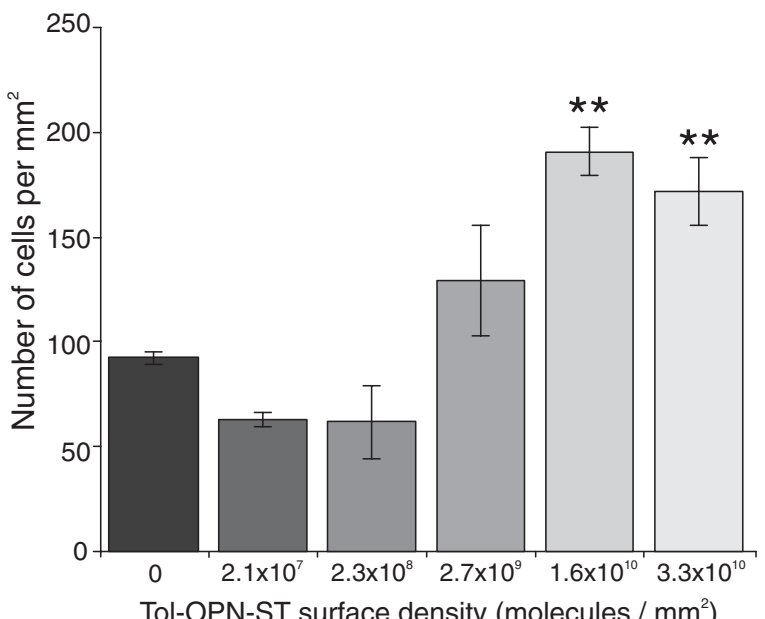

(f)

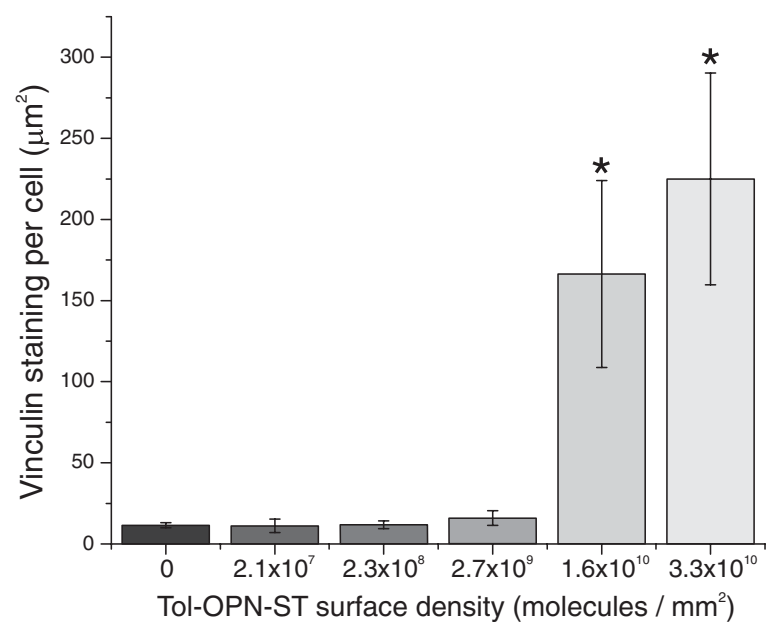

(c)

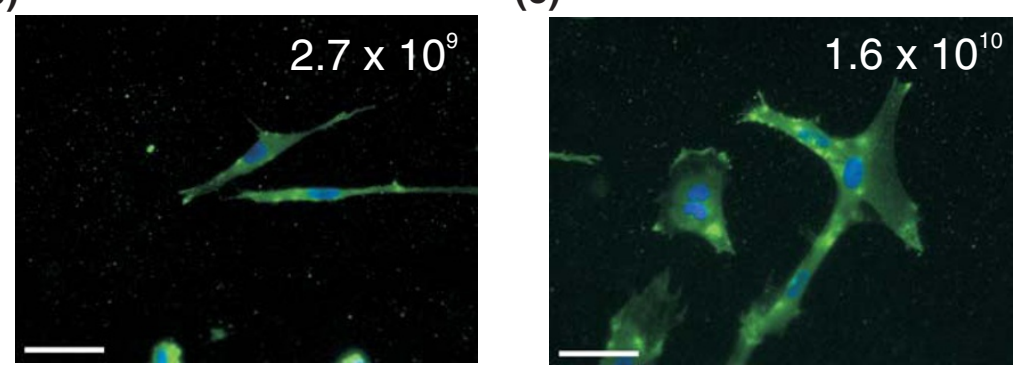

(e)

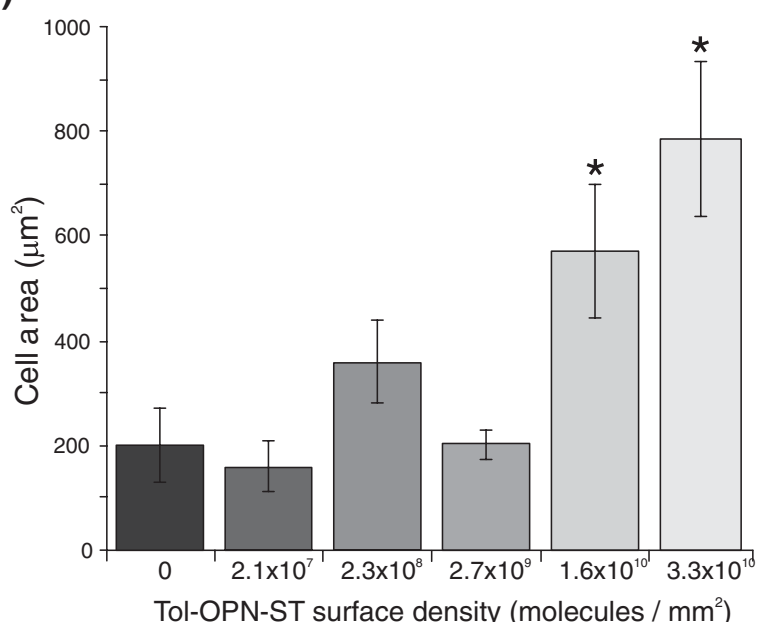

(g)

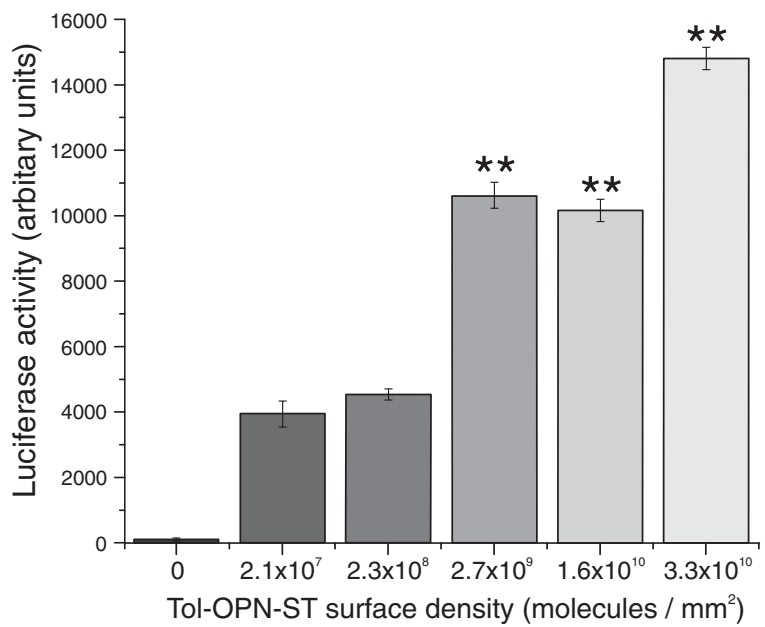

Figure 3 Tol-OP-ST surface density influences cell morphology and response to BMP2. Gold coverslips were incubated with different concentrations of Tol-OP-ST and based on SPR analysis surface densities calculated (molecules $\left./ \mathrm{mm}^{2}\right)$. (a, b, c) Cells were plated onto the surfaces, as indicated, under serum free conditions and vinculin immunolocalized 24 hours later. Size bars $=50 \mu \mathrm{m}$. (d) DAPI was used to visualize nuclear DNA and images quantified to show number of adhered cells. Numbers of cells on 1.6 and $3.3 \times 10^{10} \mathrm{molecules} / \mathrm{mm}^{2}$ were significantly different to all other surfaces. (e) Assessment of cell area revealed that cells on 1.6 and $3.3 \times 10^{10} \mathrm{molecules} / \mathrm{mm}^{2}$ were significantly different to all other surfaces. Furthermore (f) levels of vinculin positivity were significantly higher in cells on 1.6 and $3.3 \times 10^{10} \mathrm{molecules} / \mathrm{mm}^{2}$ in comparison to lower densities. (g) Cells transfected with a SMAD-reporter construct were plated on the surfaces, treated with $100 \mathrm{ng} / \mathrm{ml}$ recombinant BMP-2 and levels of luciferase assessed and normalised to cell number. Data representative of three independent experiments; results are mean $\pm \mathrm{SEM} ;{ }^{*} P<0.05$, ${ }^{* *} P<0.01$. Significance was determined by ANOVA with Fisher post test. 
response above background to the Tol protein vector alone. Next, cells transfected with the same reporter construct were plated onto specific Tol-X-ST surfaces (Figure $4 \mathrm{~b})$. Only those cells grown on the Tol-BMP-ST surface demonstrated activation of the SMAD signalling pathway that was comparable to the effect of soluble recombinant BMP. Levels of SMAD signalling observed on both TolBMP-ST surfaces and in those cells treated with recombinant BMP2 were significantly different $(P<0.01)$ compared to all other surfaces.

\section{Immobilised Tol-BMP-ST induces enhanced osteogenesis in} vitro

Tol-BMP-ST, Tol-OPN-ST and Tol-ST were then compared for their ability to influence the long term behaviour of cells in vitro. Primary rat osteoblasts can be cultured in media supplemented with $\beta$-glycerophosphate, L-ascorbic acid and dexamethasone to promote osteogenic differentiation over a period of weeks $[18,19]$. In the absence of this chemical cocktail the cells retain a more primitive fibroblastic phenotype. In our experiments, cells were maintained in culture without these soluble supplements so that the only potential osteogenic signal was from the engineered surfaces. In this environment cells proliferated and achieved confluence on all surfaces after three days. After two weeks of culture there was clear evidence of cells on Tol-BMP-ST surfaces beginning to form multilayers and discrete cellular aggregates. This behaviour was not repeated on Tol-OPN-ST, Tol-ST or other control surfaces. After four weeks, in the absence of further osteogenic factors, extensive bone-like nodule formation was observed on Tol-BMP-ST (Figure $5 \mathrm{~b}, \mathrm{~d})$ which was significantly $(P<0.01)$ more extensive than on the other surfaces investigated. There was some evidence of mineralised matrix production on Tol-OP-ST (Figure 5c) whilst on Tol-ST alone (Figure 5a) and bare gold there very few bone-like nodules (Figure $5 \mathrm{~d}$ ).

\section{Surface patterns of Tol-BMP-ST induce spatially restricted osteoblast differentiation}

Whilst osteoblasts displayed enhanced differentiation when cultured on surfaces displaying Tol-BMP-ST it was not clear if Tol-BMP-ST was acting after desorption from the surface. To address this, patterned surfaces were created using a microcontact printing approach [8]. A polydimethylsiloxane (PDMS) stamp displaying microscale features was inked with Tol-BMP-ST and used to transfer a protein pattern to the gold surface. The gaps between the protein and between patterns were backfilled by incubation of the whole surface with TEG-thiol. Osteoblasts were cultured on these surfaces for two weeks and differentiation assessed by staining for alkaline phosphatase activity (Figure 6b). During two weeks cells grew all over the surface both in regions that were stamped with the

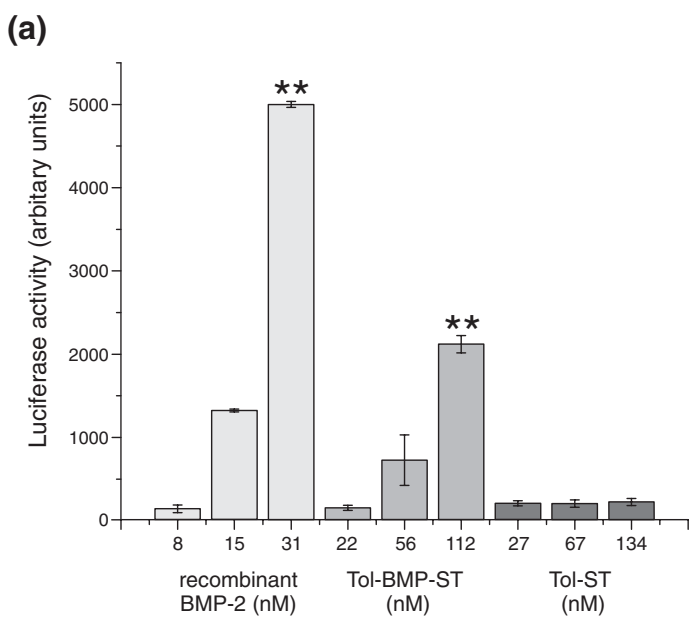

(b)

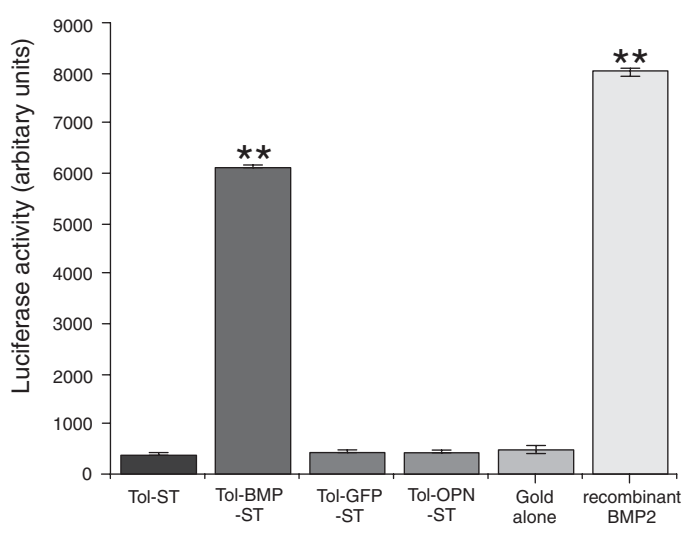

Figure 4 Tol scaffold containing the BMP2 knuckle peptide, KIPKASSVPTELSAISTLYL, activates Smad dependent signalling both when added exogenously to adherent cells and when immobilised on a surface. (a) Cells were cultured in multiwell plates, transfected with a SMAD-reporter construct and then treated with recombinant BMP2, exogenous Tol-BMP-ST or Tol-ST. Levels of luciferase were then assessed after eight hours. Both recombinant BMP2 (31 nM) and Tol-BMP-ST (112 nM) induced significant levels of luciferase activity compared to Tol alone. (b) Cells already transfected with the SMAD-reporter construct were plated onto gold coverslips displaying the scaffold proteins at the concentrations outlined in Table 1 or TEG alone. Luciferase activity was measured 12 hours later and contrasted with the same cells grown on tissue culture plastic and treated with soluble recombinant BMP-2 (100 ng/ml). Significant levels of SMAD signalling were only observed on the Tol-BMP-ST surface. Data representative of three independent experiments; results are mean \pm SEM; ${ }^{* *} P<0.01$. Significance was determined by ANOVA with Tukey post test.

Tol-BMP-ST pattern and those that were not (Figure 6a). The marker of osteoblast differentiation, alkaline phosphatase was principally localised to the regions where Tol-BMP was immobilised (Figure 6b). These experiments demonstrated that bioactive signalling was restricted to the patterned areas suggesting that surface 
(a)

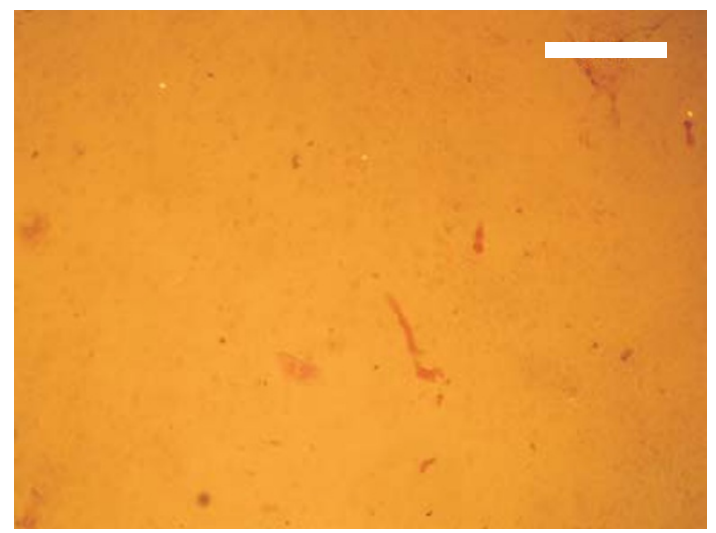

(c)

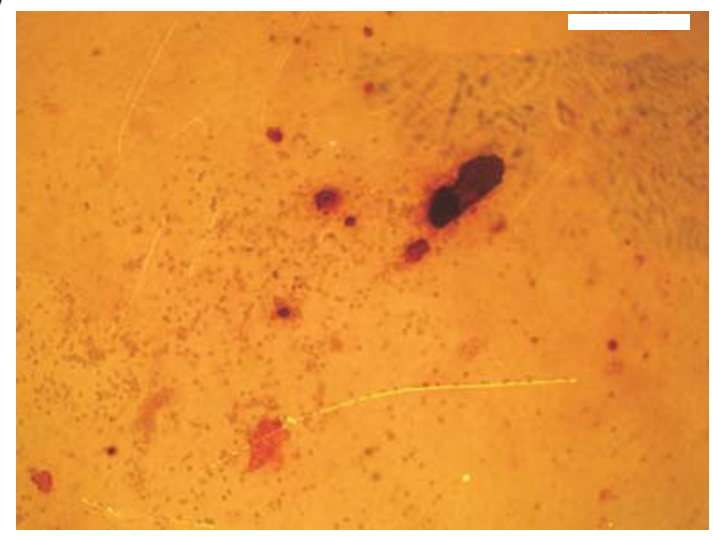

(b)

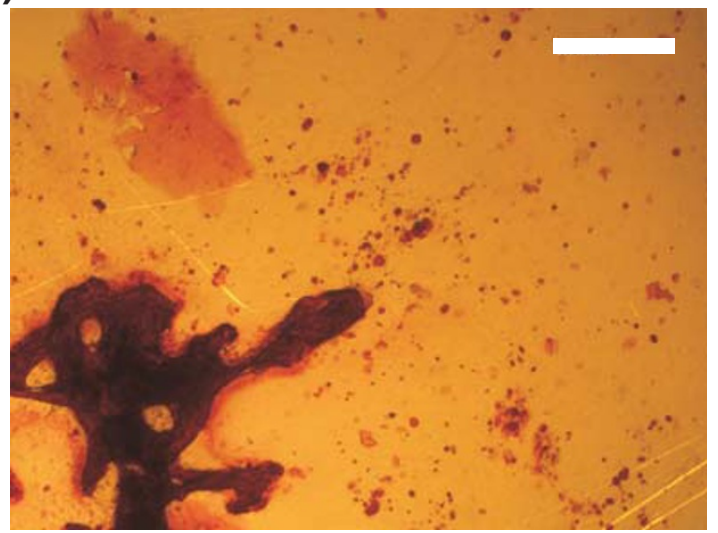

(d)

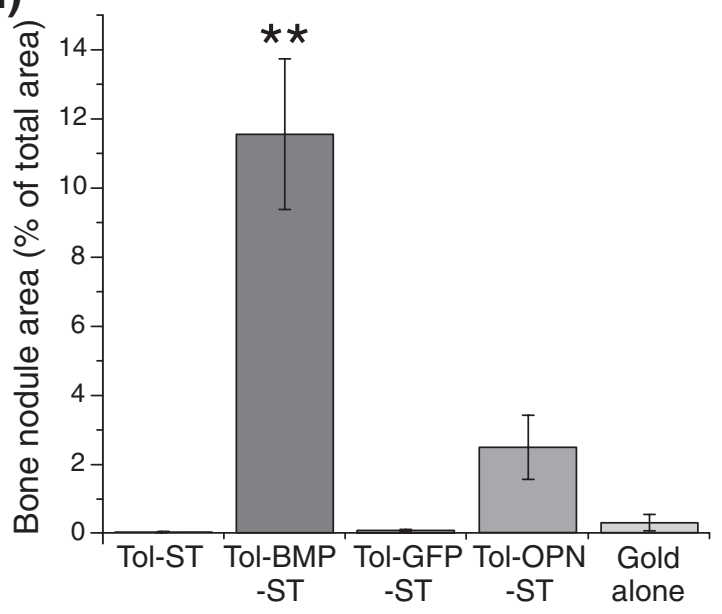

Figure 5 Long term growth of calvarial-derived cells on scaffold proteins. After four weeks under routine culture conditions and in the absence of exogeneous osteogenic factors mineralized matrix was visualized with Alizarin red staining. There was little evidence of mineralised matrix on (a) Tol alone, whilst abundant deposition was observed on (b) Tol-BMP-ST and a small amount on (c) Tol-OP-ST surfaces. Size bar $=400 \mu \mathrm{m}$. (d) Image analysis shows significant evidence of mineralised matrix deposition only on Tol-BMP surfaces. Data representative of three independent experiments; results are mean $\pm \mathrm{SEM} ;{ }^{* *} P<0.01$. Significance was determined by ANOVA with Tukey post test.

tethered ligand was predominantly responsible for the regulation of cell activity and not Tol-BMP-ST released from the surface.

\section{Discussion}

Signalling by interfacial motifs is a fundamental biological mechanism that underpins numerous aspects of morphogenesis and wound healing [1]. Tools that enable further insight into how immobilized ligands regulate cell behaviour will not only provide mechanistic understanding but also the basis for therapeutic approaches such as tissue engineering [20]. Our results show that a generic immobilisation system with a variable motif sandwiched between expression enhancing and self assembly domains (Tol-motif-ST) can present the motifs in a functional state at the surface of a SAM. The fusion protein is easily produced in large amounts in $E$ coli and is easy to convert from a highly water soluble state to a SAM inserted form on gold [8]. Analysis by SPR of the Tol-ST proteins on a gold surface interestingly provided evidence that TolOPN-ST and Tol-BMP-ST pack at higher surface densities than Tol-ST alone. This may arise from flexibility introduced into the protein by the inclusion of the short OP and BMP motifs. The highest flexibility is expected from the OP domain as there are three linker sequences found in this sequence whilst the BMP motif is shorter. In addition analysis of Tol-GFP (data not shown) assembly by SPR shows this rigid construct to be similar to Tol alone, supporting the possibility that protein flexibility plays a role in its packing on the surface. Thus retention of the $\mathrm{N}$ terminal Tol domain does not inhibit self assembly and likely serves to solubilise and protect the flexible peptides from degradation, presenting the biologically active motifs in a more constrained structure. 


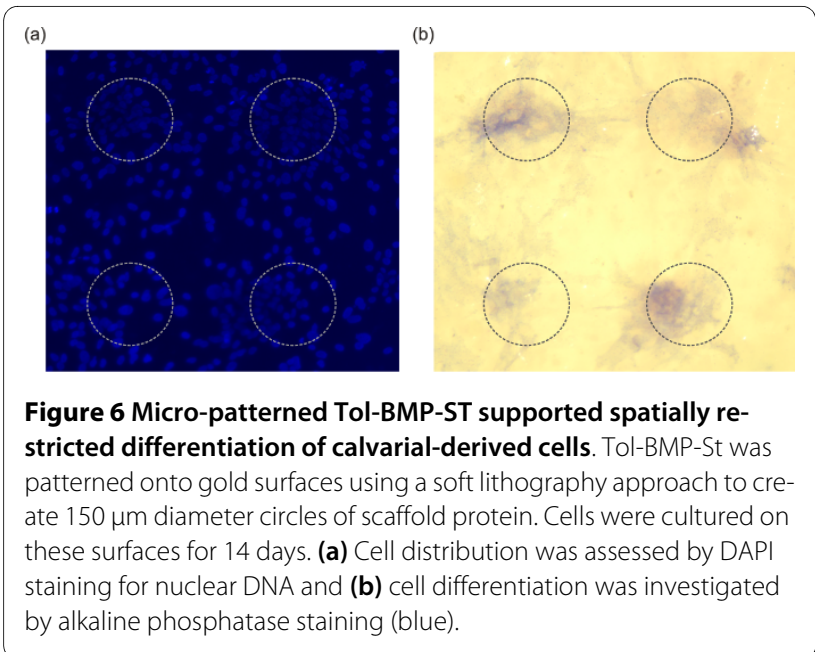

The biological activity of the Tol-ST surfaces was first determined in simple cell adhesion assays performed in the absence of serum. The abundant cell adhesion on TolOPN-ST, in contrast to the other surfaces, is most likely mediated by $\alpha_{9} \beta_{1}$ integrin as described for endothelial cells [11-14]. Calvarial derived osteoblasts have not been previously shown to express $\alpha_{9} \beta_{1}$ integrins but they can be found on periodontal ligament cells [21] and cells of the osteoclast lineage [22]. The adhesion and spreading of cells described here is a possible demonstration of $\alpha_{9} \beta_{1}$ integrins activity in these cells but an alternative mechanism cannot be discounted.

Surface assembly techniques provide the ability to control surface density of cell interaction motifs. Reducing the apparent density of Tol-OP-ST had significant effects on cell shape, morphology and numbers of vinculin-positive focal contacts. This revealed a threshold level of TolOP-ST below which cytoskeletal tensioning and remodelling cannot be supported $\left(2.7 \times 10^{9}\right.$ molecules $\left./ \mathrm{mm}^{2}\right)$, and above which the cells can interact with the surface initiating morphological change and regulation of cell behaviour. The estimated density measured here compares with other approaches that have attempted to address this issue where cells of fibroblastic origin exhibited increased cell adhesion and spreading on integrin ligands of the order $10^{9}$ molecules $/ \mathrm{mm}^{2}$ [23-25]

The way in which cells adhere and interact with the surrounding matrix is known to not only influence cell shape but also to control cell response and gene expression [17]. Indeed this has been shown to be a mechanism that is important in the regulation of osteoblast differentiation $[26,27]$ and response to BMPs [28-30]. To demonstrate that Tol-ST surfaces could be used to probe this phenomenon, different densities of Tol-OPN-ST were characterised for their ability to support BMP dependent signalling. A SMAD-responsive reporter construct showed significantly greater activation in response to
srBMP-2 when cells were cultured on Tol-OPN-ST at 2.7 $\times 10^{9}-3.3 \times 10^{10}$ molecule $/ \mathrm{mm}^{2}$ compared to $2.3 \times 10^{8}$ molecules $/ \mathrm{mm}^{2}$ and lower. It is particularly noteworthy that the apparent threshold for formation of abundant cell adhesion plaques $\left(1.6 \times 10^{10}\right.$ molecules $\left./ \mathrm{mm}^{2}\right)$ is higher than that for BMP receptor activation and signal transduction $\left(2.7 \times 10^{10}\right.$ molecules $\left./ \mathrm{mm}^{2}\right)$. This suggests that the mechanisms regulating BMP receptor signalling may well be independent of cytoskeletal organisation controlled by integrin adhesion to Tol-OPN-ST. On other substrates, $\alpha_{\mathrm{v}} \beta$ integrins [29] and Focal Adhesion Kinase (FAK) [31] have both been implicated in the osteoblastic response to BMP. Our results support general matrixdependent BMP signalling in terms of FAK activity since weakly adherent cells demonstrated poor BMP responses whilst well adhered and spread cells exhibited elevated BMP effects. Nevertheless this does not exclude a role for integrin subunit specific responses with BMPRI and BMPRII directly associating with $\alpha_{\mathrm{v}}$ and $\beta_{1}$ integrins [29], on appropriate external ECM ligands, to give altered responses.

Tol-BMP-ST maintained BMP-like activity and like srBMP-2 was able to activate the Smad signalling cascade. This is in agreement with the work of Saito et al (2003) who showed that a synthetic BMP2 knuckle peptide was able to induce alkaline phosphatase activity in $\mathrm{C} 2 \mathrm{C} 12$ cells but less effectively than srBMP2 [16]. Previous work $[32,33]$ has shown that immobilised BMP2 more effectively activates differentiation of osteoblasts than soluble BMP2. Tol-BMP-ST was immobilised on gold surfaces, back-filled with TEG-thiol and then compared with soluble recombinant BMP-2 for the ability to regulate osteoblast activity and bone formation. Immobilized Tol-BMPST showed activation of SMAD responsive elements driving luciferase expression in transfected cells. Interestingly the level of activation of signalling by the immobilised ligand was comparable to soluble recombinant BMP2, demonstrating its enhanced activity compared to soluble Tol-BMP-ST. The activity of this BMP2 knuckle peptide is postulated to be mediated through its interaction with BMP receptors I and II (BMPRI and BMPRII) [16]. Activation of preformed or induced BMPR complexes by soluble BMP results in initiation of intracellular signalling cascades that include the Smad pathway. In addition, BMPR complexes are believed to be internalised by the cell in processes which both alter the signalling dynamic and lead to loss of or attenuation of activation [34-38]. It is therefore likely that Tol-BMP-ST surfaces facilitate prolonged activation of BMP signalling since the receptor complex would be retained at the cell surface by the immobilised ligand and prevented from trafficking into the cell for example via lipid rafts. This is supported by the work of others which shows that immobilised 
recombinant BMP2 causes prolonged activation of osteogenic gene expression and enhanced osteoblast differentiation $[39,40]$.

When cells were plated on Tol-BMP-ST under routine culture conditions, cell differentiation occurred during long term experiments with evidence of the formation of abundant bone-like nodules. In contrast, few mineralised matrix deposits were observed on Tol-OPN-ST and bare gold. Immobilised BMP has previously been shown to support osteogenic differentiation in vitro $[32,40,41]$ using MC3T3-E1 cells and BMP2 that was immobilized by a heat treatment process. Data presented here use primary cells that are a heterogeneous population that retain the potential to differentiate to several different lineages but this is the first time that a tethered ligand alone and in the complete absence of other agents like dexamethasone not only induces ostegenic differentiation but also extensive deposition of a mineralized bone-like matrix. This illustrates the enhanced and sustained signalling provided by the Tol-BMP-ST surface reinforced cell regulator cues that drive the temporal control of cell phenotype. Furthermore Tol-BMP-ST on gold reproduces the asymmetric directional control of bone formation observed in $v i v o$, a process that is believed to be guided by immobilised ligands. The formation of bone-like nodules in vitro requires extensive cell motility with the formation of three-dimensional cell aggregates and therefore the immobilised signal derived from Tol-BMP-ST is likely to be relayed to cells away from the surface by cell:cell interactions and paracrine signalling.

Spatial control of osteoblast differentiation was demonstrated by using microcontact printing of Tol-BMP-ST. This approach has been used widely in cell culture studies [42] with predominantly ECM motifs but this is the first time that cell differentiation motifs have been presented in this way. This showed that it is immobilised Tol-BMP$\mathrm{SP}$ and not ligand lost from the surface that is directing cell behaviour. Work using an inkjet approach has recently reported similar findings with recombinant BMP2 spotted on a fibrin layer [43] illustrating the potential for patterns of immobilised biological ligands to control cell behaviour in a way that is spatially restricted.

\section{Conclusions}

Understanding the molecular mechanisms of cell interactions and their regulation by surrounding matrix cues has important implications for biotechnology (production of cells and cell products) and medicine (tissue engineering, prosthetic implants, cancer and developmental biology). For example, it provides the basis for fabrication of scaffolds bearing biomimetics and/or therapeutics to facilitate tight control of cell phenotype in a target tissue. The technology described here, which immobilises recombinant proteins, is a highly controlled, adaptable and repro- ducible approach that allows the creation and screening of surfaces displaying bioactive cell interaction motifs. We have shown that this approach can provide an insight into the role of immobilised ligands in regulating cell behaviour, the interplay with soluble factors and the influence of spatially controlled signalling. Furthermore, this approach presents a durable surface that allows both short (hours or days) and long term (weeks) effects on cell activity to be assessed. Therefore this technology will considerably enhance current approaches that utilise cellscaffold technology and also undoubtedly provide significant new data and therapeutic applications.

\section{Methods \\ Production of recombinant proteins in E. Coli and their immobilization on gold surfaces}

TolAIII-Switch tag fusion protein expression constructs (Tol-motif-ST) were based on the pTol-T vector, expressed in E. coli BL-21 cells and purified as detailed previously (Anderluh et al 2003). The motifs derived from osteopontin (SVVYGLRGSGSGS) and BMP-2 (KIPKASSVPTELSAISTLYL) were engineered into the expression construct using a synthetic oligonuleotide approach. The potential for recombinant proteins to interact with a gold surface was evaluated using Surface Plasmon Resonance (SPR). The gold surface was cleaned with Piranha solution for 30 minutes, rinsed with $\mathrm{H}_{2} \mathrm{O}$, $1 \%(\mathrm{w} / \mathrm{v})$ sodium dodecyl sulphate (SDS) and finally extensively with $\mathrm{H}_{2} \mathrm{O}$ before drying with air and immediate docking into the Biacore $\mathrm{X}$ system. A buffer of 200 $\mathrm{mM} \mathrm{NaCl}, 0.5 \mathrm{mM}$ EDTA, and $20 \mathrm{mM}$ Tris $\mathrm{pH} 8.0$ was used for all solutions and also as the running buffer. The solutions used for thiol binding steps were supplemented with $0.1 \mathrm{mM}$ TCEP (Tris(2-carboxyethyl)phosphine hydrochloride) for one hour before use. SPR analysis was used to calculate protein surface density (molecules/ $\mathrm{mm}^{2}$ ) based on the observation that an increase of $1 \mathrm{rU}$ equates to $1 \mathrm{pg} / \mathrm{mm}^{2}$. Molecules $/ \mathrm{mm}^{2}$ could then be found from $\mathrm{rU}$ increase $\times \mathrm{A} /\left(\mathrm{Mr}\right.$ protein $\left.\times 10^{12}\right)$, where $\mathrm{A}$ $=$ Avogadro's number. In all experiments, except where indicated, the Tol-motif-ST proteins were used to prepare surfaces with the densities outlined in Table 1. Surfaces for cell culture were prepared as above on gold-coated coverslips but were finally then immersed in $0.5 \mathrm{mM}$ TEG-thiol (11-mercapto-1-triethyleneglycolundecane, HSC11-EG3) (ProChimia Surfaces, Sopot, Poland) for four hours to complete the formation of a surface monolayer.

\section{Microcontact printing on cleaned gold coverslips}

CAD software was used to produce a five-inch mask design outlining individual $64 \mathrm{~mm}^{2}$ patterned dies. The mask was manufactured by Delta Mask Bv (Enschede, Netherlands) to a tolerance of $0.35 \mu \mathrm{m}$. A patterned sili- 
con wafer was fabricated from the mask using standard photolithographic techniques to create a photoresist master. The mold was silanized by leaving overnight in chlorotrimethylsilane vapour. Polydimethylsiloxane (Dow Corning, MIDLAND, MI, USA) was prepared as directed by the manufacturer and poured to a depth of $5 \mathrm{~mm}$ over the photoresist master, the chamber was degassed and left overnight to cure. Final cure of the PDMS was achieved by placing in an oven at $60^{\circ} \mathrm{C}$ for one hour. Stamps were cut from the PDMS layer with a scalpel and gently peeled away from the photoresist. In the experiments described here a stamp was used that created circles $150 \mu \mathrm{m}$ in diameter within a $300 \mu \mathrm{m}$ square array. The polydimethylsiloxane (PDMS) stamps were rinsed in water to remove any debris, and then immersed in ethanol and dried in a stream of compressed air, before being inked using recombinant Tol-BMP-ST $(5 \mathrm{mM})$ and then dried in a stream of compressed air. The stamps were then placed on the surface of the Piranha-treated gold, left for 15 seconds and then the gold surface allowed to dry for 10 minutes. The gold surfaces were then immersed in $0.5 \mathrm{mM}$ TEG-thiol (11-mercapto-1-triethyleneglycolundecane, HSC11-EG3) (ProChimia Surfaces, Sopot, Poland) for four hours.

\section{Cell isolation and culture}

Culture media and supplements were obtained from Invitrogen (Paisley, UK) unless otherwise stated. Primary rat osteoblasts were isolated from three-day-old rat (Sprague Dawley) calvariae (Charles River UK Ltd, Margate, Kent, UK) based on methods previously described [18]. Cells were grown in Dulbecco's modified Eagle's medium (DMEM) supplemented with $10 \%$ fetal calf serum (FCS), $100 \mu \mathrm{g} / \mathrm{ml}$ streptomycin and $100 \mathrm{U} / \mathrm{ml}$ penicillin (all Invitrogen Ltd, Paisley, UK) at $37^{\circ} \mathrm{C}$ in a humidified atmosphere with $5 \% \mathrm{CO}_{2}$. Cells were used up to passage 2 and subcultured using routine tissue culture approaches.

\section{Immunofluorescence}

Cells at 70 to $80 \%$ confluence were trypsinised and resuspended in complete media. Cells were then seeded into six well plates containing gold-coated coverslips displaying the appropriate SAMs and incubated for 24 hours at $37^{\circ} \mathrm{C}$ in a humidified atmosphere with $5 \% \mathrm{CO}_{2}$. Cells were then rinsed with PBS (Becton Dickinson UK Ltd, Oxford, UK) and fixed with $4 \%(\mathrm{w} / \mathrm{v})$ paraformaldehyde in PBS for 15 to 20 minutes. Cells were permeabilised by incubation for five minutes in $0.1 \%$ Triton X-100 (v/v) in PBS and incubated for one hour at room temperature in 1:200 dilution of monoclonal mouse anti vinculin antibody (V9131 - Sigma-Aldrich Company Ltd, Gillingham, Kent, UK)). Samples were rinsed with PBS and incubated with a FITC-goat anti-mouse antibody (Zymed Laboratories
Invitrogen Ltd, Paisley, UK). Following a wash step, cells were overlayered with VectaShield containing DAPI (Vector Laboratories Ltd, Peterborough, UK) and mounted for microscopy. The samples were then visualized on a Leica fluorescence microscope and images captured using Spot cooled CCD camera and software with digital images being analysed using ImageJ (Freeware). In analysis of cell morphology, circularity was defined as $4 \pi\left(\right.$ area/perimeter $\left.{ }^{2}\right)$.

\section{BMP responsiveness of cells}

A luciferase reporter construct that was under the transcriptional control of $6 \times$ SMAD binding elements was used to demonstrate BMP induced intracellular signalling cascade [44]. Cells were seeded overnight to 50 to $60 \%$ confluency and then transfected with the reporter construct using Fugene (Roche, Burgess Hill, UK). Cells were then either plated onto the SAMs and luciferase activity assessed at the time points indicated or transfected cells were treated with soluble recombinant protein and response investigated. Luciferase activity was measured using the Bright-Glo ${ }^{\mathrm{m}}$ Luciferase Assay System (Promega UK Ltd, Southampton, UK) and an automated microluminometer (MicroLumat Plus, Berthold Technologies (UK) Ltd, Harpenden, UK).

\section{Mineralised matrix and alkaline phosphatase staining}

Alizarin red detects the presence of calcific deposition within the matrix by binding calcium ions to form a complex that is intensely red. Cells were washed in PBS and incubated in $1 \%(\mathrm{w} / \mathrm{v})$ Alizarin red $(\mathrm{pH} 4.0)$ solution for 20 minutes in the dark before a final wash in distilled water. Cells on surfaces were then mounted onto glass slides and mounted in Vectashield with DAPI (Vector Laboratories Ltd, Peterborough, UK). To visualize alkaline phosphatase activity cell monolayers were fixed for 10 minutes in $4 \%(\mathrm{w} / \mathrm{v})$ paraformaldehyde in PBS and then incubated in napthol AS-X phosphate and Fast Blue $\mathrm{RR}$ (pH8.4).

\section{Statistical Analysis}

In all experiments data was analysed by ANOVA using either a Fisher or Tukey post test. Statistical significance was considered at ${ }^{*} P<0.05$ and ${ }^{* *} P<0.01$. Experiments are presented as representative or at least three independent experiments: for analysis of cell number $\mathrm{n}=8$; for investigation of area, circularity and vinculin staining $\mathrm{n}=$ 20; for luciferase assays $n=6$.

\footnotetext{
Abbreviations

BMP: bone morphogenetic protein; DAPI: 4,6-diamidino-2-phenylindole; DMEM: Dulbecco's modified Eagle's medium; FGF: fibroblast growth factor; IL: interleukin; OPN: osteopontin; PBS: phosphate buffered saline; PDMS: polydimethylsiloxane; SAM: self-assembled monolayer; ST: switch-tag; TCEP: Tris(2-carboxyethyl)phosphine hydrochloride; TEG: triethyleneglycolundecane; TGF: transforming growth factor; VEGF: vascular endothelial growth factor.
} 


\section{Authors' contributions}

$J H L, A W M$ and MAB conceived the study and designed the experiments. BTC designed and engineered the constructs. EAM performed all the experiments and drafted the manuscript. All authors edited, read and approved the final manuscript.

\section{Acknowledgements}

The authors are grateful to the BBSRC for the support of BTC (Grant 13/E19051) and EAM.

\section{Author Details}

'Institute for Cellular Medicine, The Medical School, Newcastle University, Newcastle-upon-Tyne, NE2 4HH, UK and Institute for Cell and Molecular Biosciences, The Medical School, Newcastle University, Newcastle-upon-Tyne, NE2 $4 \mathrm{HH}, \mathrm{UK}$

Received: 18 December 2009 Accepted: 10 May 2010

Published: 10 May 2010

\section{References}

1. Daley WP, Peters SB, Larsen M: Extracellular matrix dynamics in development and regenerative medicine. J Cell Sci 2008, 121:255-264.

2. Pierschbacher MD, Ruoslahti E: Variants of the cell recognition site of fibronectin that retain attachment-promoting activity. Proc Natl Acad Sci USA 1984, 81:5985-5988.

3. Humphries MJ, Obara M, Olden K, Yamada KM: Role of fibronectin in adhesion, migration, and metastasis. Cancer Invest 1989, 7:373-393.

4. Garamszegi N, Garamszegi SP, Shehadeh LA, Scully SP: Extracellular matrix-induced gene expression in human breast cancer cells. Mol Cancer Res 2009, 7:319-329.

5. Sahni A, Sporn LA, Francis CW: Potentiation of endothelial cell proliferation by fibrin(ogen)-bound fibroblast growth factor-2. J Biol Chem 1999, 274:14936-14941.

6. Ehrbar M, Metters A, Zammaretti P, Hubbell JA, Zisch AH: Endothelial cell proliferation and progenitor maturation by fibrin-bound VEGF variants with differential susceptibilities to local cellular activity. $J$ Control Release 2005, 101:93-109.

7. Sahni A, Guo M, Sahni SK, Francis CW: Interleukin-1 beta but not IL1alpha binds to fibrinogen and fibrin and has enhanced activity in the bound form. Blood 2004, 104:409-414.

8. Chaffey BT, Mitchell E, Birch MA, Lakey JH: A generic expression system to produce proteins that co-assemble with alkane thiol SAM. Int J Nanomedicine 2008, 3:287-93.

9. Shah DS, Thomas MB, Phillips S, Cisneros DA, Le Brun AP, Holt SA, Lakey $\mathrm{JH}$ : Self-assembling layers created by membrane proteins on gold. Biochem Soc Trans 2007, 35:522-526.

10. Anderluh G, Gokce I, Lakey JH: Expression of proteins using the third domain of the Escherichia coli periplasmic-protein TolA as a fusion partner. Protein Expr Purif 2003, 28:173-181.

11. Yokosaki Y, Matsuura N, Sasaki T, Murakami I, Schneider H, Higashiyama S, Saitoh Y, Yamakido M, Taooka Y, Sheppard D: The Integrin $\alpha_{9} \beta_{1}$ bind to a novel recognition sequence (SVVYGLR) in the thrombin-cleaved amino-terminal fragment of osteopontin. J Biol Chem 1999, 274:36328-36334

12. Hamada Y, Nokihara K, Okazaki M, Fujitani W, Matsumoto T, Matsuo M, Umakoshi Y, Takahashi J, Matsuura N: Angiogenic activity of osteopontin-derived peptide SVVYGLR. Biochem Biophys Res Commun 2003, 310:153-157.

13. Hamada Y, Yuki K, Okazaki M, Fujitani W, Matsumoto T, Hashida MK, Harutsugu K, Nokihara K, Daito M, Matsuura N, Takahashi J: Osteopontinderived peptide SVVYGLR induces angiogenesis in vivo. Dent Mater J 2004, 23:650-655.

14. Hamada Y, Egusa H, Kaneda Y, Hirata I, Kawaguchi N, Hirao T, Matsumoto T, Yao M, Daito K, Suzuki M, Yatani H, Daito M, Okazaki M, Matsuura N: Synthetic osteopontin-derived peptide SVVYGLR can induce neovascularization in artificial bone marrow scaffold biomaterials. Dent Mater J 2007, 26:487-492.

15. Saito A, Suzuki Y, Kitamura M, Ogata S, Yoshihara Y, Masuda S, Ohtsuki C, Tanihara M: Repair of 20-mm long rabbit radial bone defects using BMP-derived peptide combined with an alpha-tricalcium phosphate scaffold. J Biomed Mater Res A 2006, 77:700-706.
16. Saito A, Suzuki Y, Ogata S, Ohtsuki C, Tanihara M: Activation of osteoprogenitor cells by a novel synthetic peptide derived from the bone morphogenetic protein-2 knuckle epitope. Biochim Biophys Acta 2003, 1651:60-67.

17. Streuli $\mathrm{CH}$, Akhtar N: Signal co-operation between integrins and other receptor systems. Biochem J 2009, 418:491-506.

18. Bokhari MA, Akay G, Zhang S, Birch MA: The enhancement of osteoblast growth and differentiation in vitro on a peptide hydrogel - PolyHIPE Polymer hybrid material. Biomaterials 2005, 26:5198-5520.

19. Kirmizidis G, Birch MA: Microfabricated Grooved Substrates Influence Cell-Cell Communication and Osteoblast Differentiation In Vitro. Tissue Eng Part A 2009, 15:1427-1436.

20. Place ES, Evans ND, Stevens MM: Complexity in biomaterials for tissue engineering. Nat Mater 2009, 8:457-470.

21. Lallier TE, Yukna R, Moses RL: Extracellular matrix molecules improve periodontal ligament cell adhesion to anorganic bone matrix. J Dent Res 2001, 80:1748-1752.

22. Rao H, Lu G, Kajiya H, Garcia-Palacios V, Kurihara N, Anderson J, Patrene K, Sheppard D, Blair HC, Windle JJ, Choi SJ, Roodman GD: Alpha9beta1: a novel osteoclast integrin that regulates osteoclast formation and function. J Bone Miner Res 2006, 21:1657-1665.

23. Irvine DJ, Ruzette AV, Mayes AM, Griffith LG: Nanoscale clustering of RGD peptides at surfaces using comb polymers. 2. Surface segregation of comb polymers in polylactide. Biomacromolecules 2001, 2:545-556

24. Koo LY, Irvine DJ, Mayes AM, Lauffenburger DA, Griffith LG: Co-regulation of cell adhesion by nanoscale RGD organization and mechanical stimulus. J Cell Sci 2002, 115:1423-1433.

25. Arnold M, Cavalcanti-Adam EA, Glass R, Blümmel J, Eck W, Kantlehner M, Kessler $\mathrm{H}$, Spatz JP: Activation of integrin function by nanopatterned adhesive interfaces. Chemphyschem 2004, 5:383-388.

26. Takeuchi Y, Suzawa M, Kikuchi T, Nishida E, Fujita T, Matsumoto T: Differentiation and transforming growth factor- $\beta$ receptor downregulation by collagen- $\alpha 2 \beta 1$ integrin interaction is mediated by focal adhesion kinase and its downstream signals in murine osteoblastic cells. J Biol Chem 1997, 272:29309-29316.

27. Pallu S, Bareille R, Dard M, Kessler H, Jonczyk A, Vernizeau M, AmédéeVilamitjana J: A cyclo peptide activates signaling events and promotes growth and the production of the bone matrix. Peptides 2003, 24:1349-1357

28. Jikko A, Harris SE, Chen D, Mendrick DL, Damsky CH: Collagen integrin receptors regulate early osteoblast differentiation induced by BMP-2. J Bone Miner Res 1999, 14:1075-1083.

29. Lai C-F, Cheng S-L: av $\beta$ integrins play an essential role in BMP-2 induction of osteoblast differentiation. J Bone Miner Res 2005, 20:330-340

30. Marquis ME, Lord E, Bergeron E, Bourgoin L, Faucheux N: Short-term effects of adhesion peptides on the responses of preosteoblasts to pBMP-9. Biomaterials 2008, 29:1005-1016.

31. Tamura Y, Takeuchi Y, Suzawa M, Fukumoto S, Kato M, Miyazono K, Fujita T: Focal adhesion kinase activity is required for bone morphogenetic protein--Smad1 signaling and osteoblastic differentiation in murine MC3T3-E1 cells. J Bone Miner Res 2001, 16:1772-1779.

32. Wiemann M, Jennissen HP, Rumpf H, Winkler $L$, Chatzinikolaidou M, Schmitz I, Bingmann D: A reporter-cell assay for the detection of BMP-2 immobilized on porous and nonporous materials. J Biomed Mater Res 2002, 62:119-127.

33. Matsuzaka K, Yoshinari M, Kokubu E, Shimono M, Inoue T: Behavior of osteoblast-like cells on Fibronectin or BMP-2 immobilized surface. iomed Res 2004, 25:B263-268.

34. Gilboa L, Nohe A, Geissendorfer T, Sebald W, Henis YI, Knaus P: Bone morphogenetic protein receptor complexes on the surface of live cells: a new oligomerization mode for serine/threonine kinase receptors. Mol Biol Cell 2000, 11:1023-1035.

35. Nohe A, Keating E, Underhill TM, Knaus P, Petersen NO: Effect of the distribution and clustering of the type I A BMP receptor (ALK3) with the type II BMP receptor on the activation of signalling pathways. J Cell Sci 2003, 116:3277-3284

36. Hartung A, Bitton-Worms $K$, Rechtman MM, Wenzel V, Borgermann JH, Hassel S, Henis YI, Knaus P: Different routes of BMP receptor endocytosis influence BMP signaling. Mol Cell Biol 2006, 26:7791-7805.

37. Singhatanadgit W, Mordan N, Salih V, Olsen I: Changes in bone morphogenetic protein receptor-IB localisation regulate osteogenic 
responses of human bone cells to bone morphogenetic protein-2. Int J Biochem Cell Biol 2008, 40:2854-2864.

38. Tsujigiwa H, Nagatsuka H, Lee YJ, Han PP, Gunduz M, Legeros RZ, Inoue M, Yamada M, Nagai N: Immobilized rhBMP-2/succinylated type I atelocollagen gene expression of intracellular signaling molecules on ST2 cells. J Biomed Mater Res A 2006, 77:507-511.

39. Yamachika E, Tsujigiwa H, Shirasu N, Ueno T, Sakata Y, Fukunaga J, Mizukawa N, Yamada M, Sugahara T: Immobilized recombinant human bone morphogenetic protein-2 enhances the phosphorylation of receptor-activated Smads. J Biomed Mater Res A 2009, 88:599-607.

40. Winkler L, Bingmann D, Wiemann M: Heat treatment of BMP-2 depots on implant materials generates an immobilized layer of BMP-2 with pronounced bioactivity. J Biomed Mater Res A 2006, 79:895-901.

41. Lim TY, Wang W, Shi Z, Poh CK, Neoh KG: Human bone marrow-derived mesenchymal stem cells and osteoblast differentiation on titanium with surface-grafted chitosan and immobilized bone morphogenetic protein-2. J Mater Sci Mater Med 2009, 20:1-10.

42. Falconnet D, Csucs G, Grandin HM, Textor M: Surface engineering approaches to micropattern surfaces for cell-based assays. Biomaterials 2006, 27:3044-3063.

43. Phillippi JA, Miller E, Weiss L, Huard J, Waggoner A, Campbell P: Microenvironments engineered by inkjet bioprinting spatially direct adult stem cells toward muscle- and bone-like subpopulations. Stem Cells 2008, 26:127-134.

44. Kusanagi K, Inoue H, Ishidou Y, Mishima HK, Kawabata M, Miyazono K: Characterization of a bone morphogenetic protein-responsive Smadbinding element. Mol Biol Cell 2000, 11:555-565.

doi: 10.1186/1741-7007-8-57

Cite this article as: Mitchell et al., Controlled spatial and conformational display of immobilised bone morphogenetic protein-2 and osteopontin signalling motifs regulates osteoblast adhesion and differentiation in vitro $B M C$ Biology 2010, 8:57

Submit your next manuscript to BioMed Centra and take full advantage of:

- Convenient online submission

- Thorough peer review

- No space constraints or color figure charges

- Immediate publication on acceptance

- Inclusion in PubMed, CAS, Scopus and Google Scholar

- Research which is freely available for redistribution

Submit your manuscript at www.biomedcentral.com/submit
C Biomed Central 